\title{
TEM study of structure of graphene layers in shungite carbon
}

\author{
V. V. Kovalevski, I. A. Moshnikov \\ Institute of Geology, Karelian Research Center, RAS, Petrozavodsk, Russia \\ kovalevs@krc.karelia.ru, igorm@krc.karelia.ru
}

PACS 68.37.0g, 61.48.-c

DOI 10.17586/2220-8054-2016-7-1-210-213

\begin{abstract}
Shungites have been studied by high-resolution transmission electron microscopy (HRTEM). Two types of carbon layers have been revealed in shungites by HRTEM. The first type, graphite-like carbon layers are characterized by strongly marked hexagonal symmetry. The second type are remarkable for imperfections connected with insignificant disorder in the direction of fringes and the distance between them, and can result from point defects or the pentagonal and heptagonal carbon rings that are signs of fullerene-like structures.
\end{abstract}

Keywords: Shungite, High-resolution electron microscopy, Molecular structure.

Received: 20 November 2015

\section{Introduction}

One of the more intriguing types of non-crystalline carbon occurs in the shungite rocks of Karelia (Russia), from which the first natural occurrence of fullerenes was reported [1] and hollow nanospheres and fibers were described [2]. Shungite rocks are remarkable for very complex genesis defined by an alternation of sedimentary and volcanic processes with diabase and hydrothermal intrusions [3]. There is no consensus in the literature regarding the origin of shungite rocks. Many hypotheses have been proposed, including biogenic, metasomatic, metamorphic and volcanogenic modes of origin together or separately. Shungite rocks are natural composite materials with interpenetrating carbon and mineral matrices. Carbon from shungite rocks is of the non-graphitizable variety and is characterized by turbostratic stacking of the carbon layers. Furthermore, carbon from shungite rocks demonstrates some fullerene-like characteristics on different structural and physical levels. For example, a distinctive feature of some shungites is the considerable increase in the diamagnetism at liquid-nitrogen temperatures with a temperature range and amount of change similar to that of $\mathrm{CuC}_{60}$ [4]. The effect is likely sensitive to the structure of the carbon layers and the presence of trace elements and has not been reported for amorphous natural carbon and graphite but has been observed for doped fullerites. All types of shungite rocks have variable physico-chemical properties depending on the shungite structure, $\mathrm{C}$ content, the composition and characteristics of minerals and distribution of carbon and minerals. Unfortunately, there are no direct structural data sustaining an occurrence of faultiness of carbon layers in shungites. The goal of this study is to clarify structural features of carbon layers of the natural carbon.

\section{Experimental and results}

A JEOL 4000 EX and HB-5 electron microscopes were used for the high-resolution electron microscopy (HRTEM) and for nanodiffraction study [5]. Samples of shungites from Nigozero, Shunga, Maksovo, Zazhogino and Chebolaksha deposits were powdered in water and precipitated onto lacey carbon films placed on copper grids. Images were recorded photographically and processed, as reported elsewhere [5]. 
All high-resolution transmission electron microscopy (HRTEM) images of shungites contain well-defined fringes, and these occur in packets of 5 to 14 sheets (Fig. 1). Those suggest that some 3-dimensional closed shells occur but, more commonly, there are fractions of such shells or regions of graphene structure that are highly disordered into short bent stacks and enveloped nano-sized pores. Some graphene layers form irregular closed shells, similar to those in carbon nanotubes [5]. A number of nanodiffraction patterns obtained in the stationary beam with a diameter of $0.7 \mathrm{~nm}$, contains several spots corresponding to the distance of $0.34 \mathrm{~nm}$, indicating the presence in the illuminated areas of packages consisting of graphene layers that are almost parallel to the incident beam. For some areas, the obtained nanodiffraction pictures with hkl reflections that meet the 3-dimensional order in the packing of layers, including, for example, hexagonal (ABABA..) and rhombohedral (ABCABC..) graphite [5]. However, their hkl where these reflexes are observed, are weak, diffuse, and are parallel to the 001 reflections, which would seem to indicate poorly structured packing layers. In general, for shungites, there are typical nanodiffraction patterns from graphene layers, approximately perpendicular to the incident beam and containing, reflexes, close to the 100 and 110 reflections of graphite, i.e. azimuthal disordered layers.

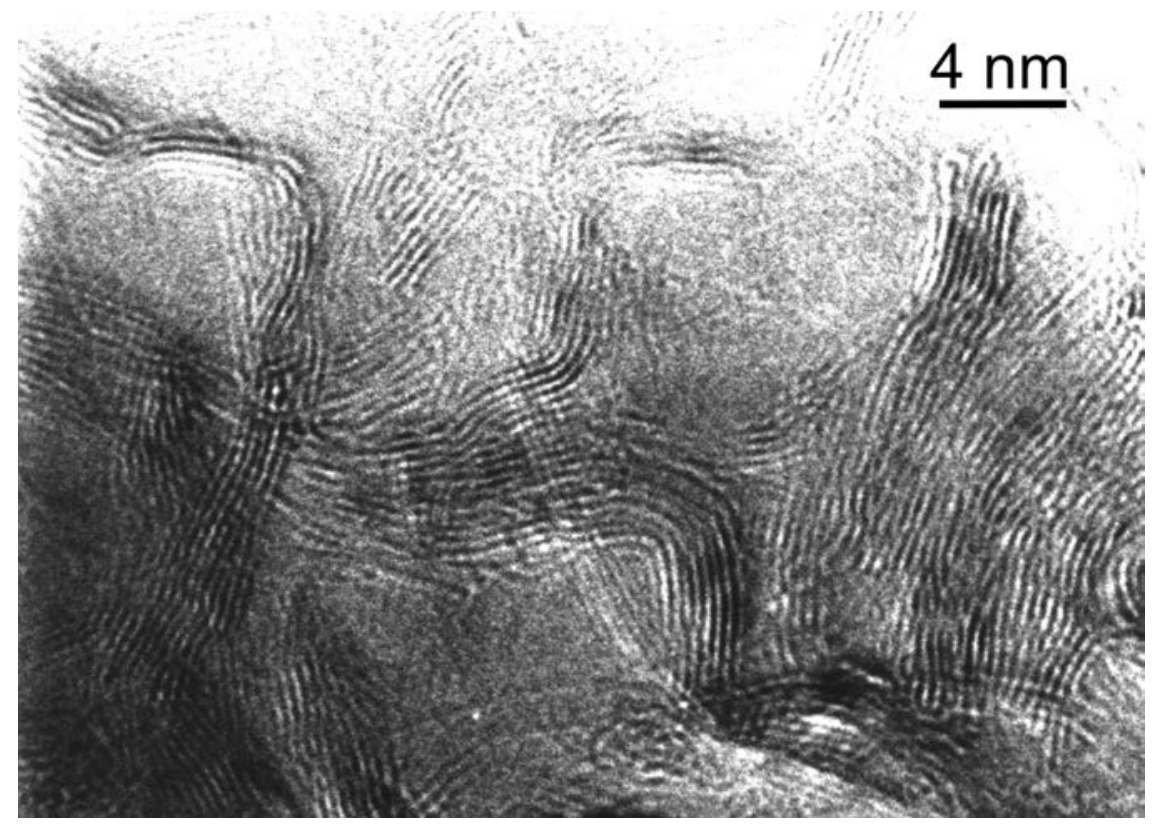

FIG. 1. HRTEM image of a thin region at the edge of Zazhogino shungite

No single extended carbon layers, which could represent graphenes were found. The exception to this were smoothly curved short-length layers up to $10 \mathrm{~nm}$, forming open and closed structures, including, similar in configuration and size, fullerenes $\mathrm{C}_{240}$ and $\mathrm{C}_{560}$ (Fig. 2) [6].

Two types of carbon layers have been revealed in shungites by HRTEM (Fig. 3). Note that both types of layers in Fig. 3 form packets including at least eight and three layers. The first type, graphite-like carbon layers are characterized by strongly marked hexagonal symmetry, but are corrugated layers. The layers consist of domains about $1 \mathrm{~nm}$ in diameter, some of those are elevated and form hillocks and others are down and form hollows. The distance between hillocks and hollows is about $2 \mathrm{~nm}$ (left image in Fig. 3).

Imperfections of the latter type of carbon layers on the molecular level connected with irregularities of 100 fringes that suggest a disorder of the carbon layers from HRTEM images. Some of those are connected with essential disorder of fringes that may result from the presence 


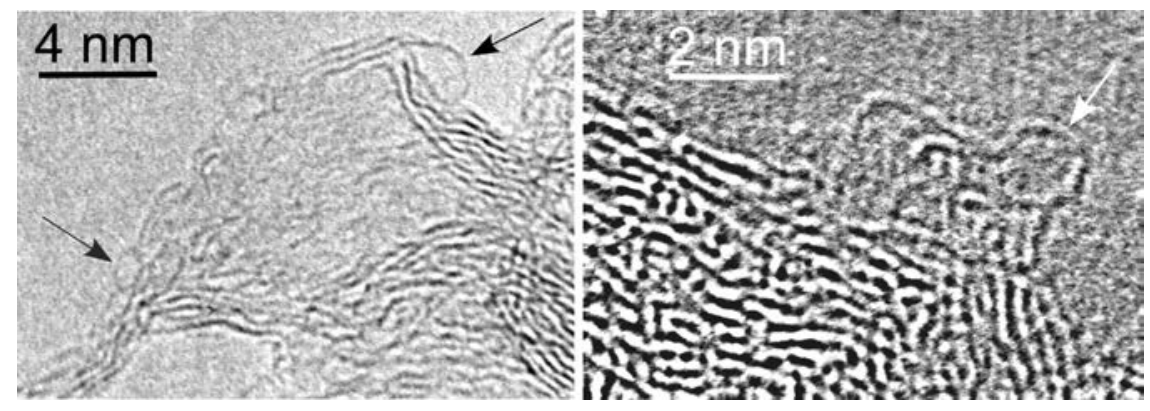

FIG. 2. HRTEM images of a thin regions at the edge of Chebolaksha (left) and Maksovo shungites. The left figure demonstrates smoothly curved short-length carbon layers (arrowed), right one shows objects (arrowed) that are similar in form and size to higher fullerenes

of impurity atoms or clusters in the stack of carbon layers (i in Fig. 3). Other imperfections connected with insignificant disorder in the direction of fringes and the distance between them are a result of the pentagonal and heptagonal carbon rings. Those can be deduced from the electron density distribution and the number of adjacent carbon rings bonded with the pentagonal and heptagonal ones ( $\mathrm{p}$ and $\mathrm{h}$ respectively in Fig. 3). The change of the electron microscopy contrast testifies to the effect that the layers are also corrugated.
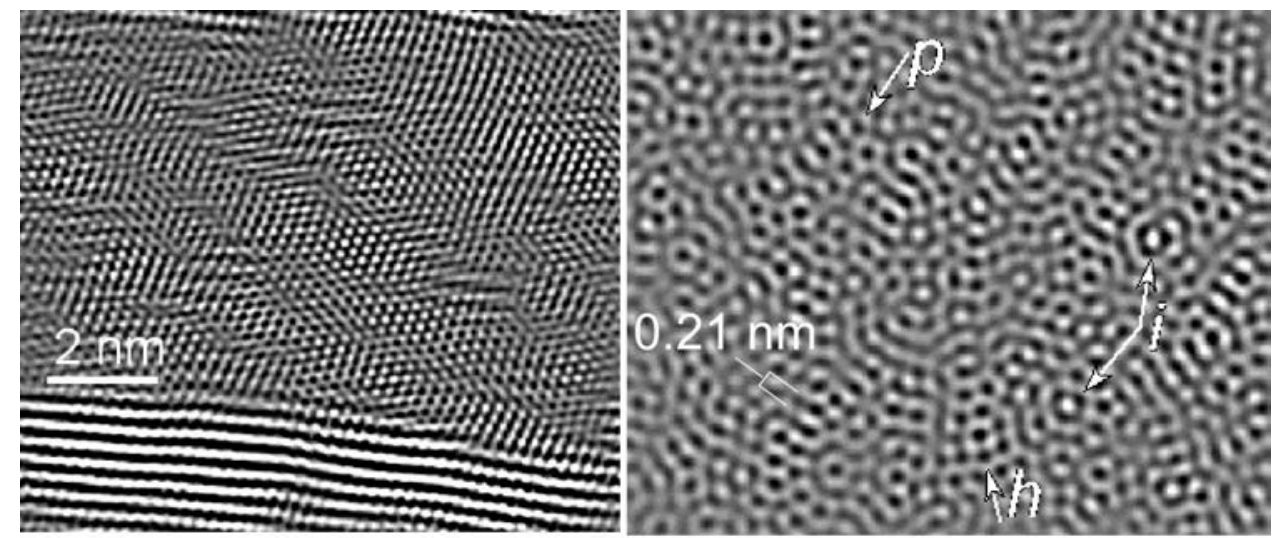

FIG. 3. HRTEM images (after Fourier filtration) of graphite-like (left) and fullerene-like carbon layers ( $\mathrm{p}$ - pentagonal, $\mathrm{h}$ - heptagonal carbon rings, $\mathrm{i}$ noncarbon inclusions) in Maksovo shungite

\section{Discussion}

HRTEM studies have shown that in shungites, usually 5 to 14 carbon layers are stacked together with $3.4 \AA$ spacing, but no single extended carbon layers were observed at all. There are short-length layers, forming open and closed nano-sized structures, among them similar in configuration and size to higher fullerenes. Extended carbon layers forming packets in shungites are corrugated to a greater or lesser extent. Two types of the carbon layers have been established in shungites by HRTEM. Carbon layers of the first type are characterized by clear two-dimensional hexagonal symmetry without any significant amount of impurity atoms and may be specified as graphite-like ones. It should be noted that the corrugation leads to an increase of the cohesive energy between adjacent carbon layers owing to the formation of potential wells and may result in the characteristic properties of graphene quantum dots [7]. Carbon layers 
of the latter type consist of not only hexagonal but also pentagonal and heptagonal carbon rings that are signs of fullerene-like structures [8]. Thus, those may be specified as fullerine-like carbon layers. The left Fig. 3 image offers no a single carbon layer but a packet enclosed three layers. Additionally, the HRTEM image of those demonstrates some clusters that have essentially more electron scattering then an ambient carbon layer and therefore might contain metal atoms. After the Fourier filtration, the clusters present as isolated contrasting dots (i in Fig. 3) that on the basis of their sizes are not only the intralayer, but also interlaminar defects. The clusters also change the band structure of shungites and may alter some physicochemical properties $[4,7]$.

\section{Acknowledgements}

This work was supported in part by the RFBR grant 13-05-98811.

\section{References}

[1] Buseck P.R., Tsipursky S.J., Hettich R. Fullerenes from the geological environment. Science, 1992, 257, P. 215-217.

[2] Kovalevski V.V., Safronov A.N., Markovski Ju.A. Hollow carbon microspheres and fibres produced by catalytic pyrolysis and observed in shungite rocks. Mol Mater., 1996, 8, P. 21-24.

[3] Chazhengina S.Y., Kovalevski V.V. Structural characteristics of shungite carbon subjected to contact metamorphism overprinted by greenschist-facies regional metamorphism. Eur. J. Mineral., 2013, 25, P. 835-843.

[4] Kovalevski V.V., Prikhodko A.V., Buseck P.R. Diamagnetism of natural fullerene-like carbon. Carbon, 2005, 43(2), P. 401-405.

[5] Kovalevski V.V., Buseck P.R., Cowley J.M. Comparison of carbon in shungite rocks to other natural carbons: An X-ray and TEM study. Carbon, 2001, 39, P. 243-256.

[6] Mordkovich V.Z. The observation of large concentric shell fullerenes and fullerene-like nanoparticles in laser pyrolysis carbon blacks. Chem. Mater., 2000, 12, P. 2813-2818.

[7] Razbirin B.S., Rozhkova N.N., Sheka E.F., Nelson D.K., A.N. Starukhin, A. S. Goryunov. Spectral properties of shungite quantum dots. Nanosystems: Phys., Chem., Math., 2014, 5 (2), P. 217-233.

[8] Cataldo F. The impact of a fullerene-like concept in carbon black science. Carbon, 2002, 40(2), P. 157-162. 